International Journal of Anesthesiology \& Research (IJAR)

ISSN 2332-2780

\title{
I-Gel and Classic LMA - a Comparitive Study in Anaesthetized and Paralyzed Patients
}

\author{
Review Article
}

Joshi $\mathrm{K}^{1^{*}}$, Kane $\mathrm{D}^{2}$, Umbarkar $\mathrm{S}^{3}$

${ }^{1}$ P.G. Student, Department of Anaesthesiology, T N Medical College \& B Y L Nair Hospital, Mumbai, India.

${ }^{2}$ Professor, Department of Anaesthesiology, Seth GSMC and KEM Hospital, Parel, Mumbai, India.

${ }^{3}$ Professor, Department of Anaesthesiology, Seth G.S. Medical College, Mumbai, Maharashtra, India.

\begin{abstract}
This study was undertaken in 50 ASA 1 and 2 patients of age group 18 to 60 years undergoing elective surgeries of short duration. Patients were randomly divided into 2 groups of 25 each for i-gel and classic LMA. Parameters assessed were ease of insertion of airway device i.e. no. of attempts required and time taken for effective airway establishment (TFEA), ease of gastric tube placement, gastric insufflation and regurgitation (by comparing $\mathrm{pH}$ of the secretions on the dorsal surface of both the devices with sensitive litmus paper), hemodynamic response to insertion of device and post-operative sore throat following general anaesthesia. Incidents like desaturation, laryngospasm, bronchospasm, gastric insufflations were noted. The mode of induction, maintenance and reversal of general anaesthesia remained the same for both the groups. Demographic data between the two groups i.e. age, weight, sex, duration of surgery was comparable and with no statistical difference between them.
\end{abstract}

There was one incidence of failure of insertion with Classic LMA to achieve effective airway. Success rate of device insertion was higher for i-gel than classic LMA and was statistically significant. Mean attempt for i-gel was 1.12 (S.D 0.332), classic LMA 1.45 (S.D 0.588). Time required for effective airway establishment was shorter with i-gel 20.24 seconds (S.D 22.408) than classic LMA 52.458 (S.D 41.341) and was statistically significant $\mathrm{P}<0.05$. There were no statistical and clinical differences in hemodynamic responses to insertion of both the devices. After removal at the end of surgery blood was visible on cuffs of 3 Classic LMAs but not on any of the i-gels. No statistical difference was found in $\mathrm{pH}$ of the cuffs of both the devices after removal. Incidence of post-operative sore throat was more with classic LMA (mean rank 31.25) than i-gel (mean rank 19) and was statistically significant $\mathrm{P}<0.05$.

To conclude i-gel is easier to insert than Classic LMA and also facilitates gastric tube placement. i-gel is appropriate device for airway management as an alternative or backup device to Classic LMA. i-gel is less traumatic to airway than Classic LMA and correctly positioned i-gel isolates glottis from upper esophagus and provides airway protection.

\author{
*Corresponding Author: \\ Kunal Joshi, \\ P.G. Student, Department of Anaesthesiology, T N Medical College \& \\ B Y L Nair Hospital, Mumbai, India. \\ Tel: +919769027806 \\ E-mail: drkunaljoshi2000@gmail.com
}

Received: March 12, 2015

Accepted: June 04, 2015

Published: June 13, 2015

Citation: Joshi K, Kane D, Umbarkar S (2015) I-Gel and Classic LMA - a Comparitive Study in Anaesthetized and Paralyzed Patients. Int J Anesth Res. 3(4), 109-112. doi: http://dx.doi.org/10.19070/2332-27801500028

Copyright: Joshi $\mathbf{K}^{\odot}$ 2015. This is an open-access article distributed under the terms of the Creative Commons Attribution License, which permits unrestricted use, distribution and reproduction in any medium, provided the original author and source are credited.

\section{Introduction}

The Laryngeal Mask Airway [LMA] was invented by Archie Brain in 1981 it first became commercially available in 1988 [1]. Since then it is frequently used in many centres. Initially the LMA was used mostly during general anaesthesia with spontaneous ventilation but a survey in United Kingdom revealed that $44 \%$ of the patients underwent positive pressure ventilation using the LMA [2]. Stated advantages of LMA with respect to tracheal intubation include absence of need for muscle relaxants and decreased risk of post-operative sore throat [3].

Since the discovery of the classic LMA many different designs of other supra-glottic airway devices were invented like proseal LMA, flexible LMA, LMA supreme etc. A potential risk of LMA is an incomplete mask seal causing gastric insufflations or oropharyngeal air leak [4]. A new variant of supra-glottic airway device i-gel was invented in January 2007 in London by Dr. Nasir. It contained second channel as gastric vent placed lateral to the airway channel intended to separate the alimentary and respiratory tracts. It permits escape for the gastric contents and reduces risk of regurgitation and pulmonary aspiration. It can also determine the correct positioning of airway.

The unique feature of i-gel is the non-inflatable cuff made of gel like material called SEBS ( Styrene Ethylene Butene Styrene). This soft gel like material reduces the risk of post-operative sore 
throat as there is minimal soft tissue compression. The cuff is mirror image of supra-glottic structures. It anatomically fits into the supra-glottic pharyngeal space. Many more features like gastric channel, epiglottic blocker, integral bite block, buccal cavity stabiliser make i-gel the unique supra-glottic airway device. As igel has firm tube section there is no need of inserting the finger in the oral cavity while placing the i-gel hence the ease of insertion is also improved. Buccal cavity stabiliser is laterally flattened, elliptical and firm that gives it stability after insertion. As there is no cuff inflation there are less chances of displacement. i-gel is also a latex free device.

In this prospective study we compared the techniques with respect to ease of insertion of the airway device i.e. LMA Classic and i-gel, ease of insertion of naso-gastric tube for i-gel, hemodynamic changes, incidence of regurgitation and post-operative sore throat complaints.

\section{Methods}

The present prospective randomized study was conducted in B.Y.L. Nair Hospital, Mumbai in the Department of Anaesthesia after obtaining ethics committee clearance and written informed consent. Fifty ASA I and II patients of either sex posted for elective general surgeries like hernioplasties, breast lump excision, skin grafting, axillary lymph node excision, fistula in ano, lipoma excision were included in the study. Patients with difficult airway, full stomach, gastro-esophageal reflux, pregnancy, post-radiotherapy to neck, smokers were excluded from the study.

All patients received iv Glycopyrolate $4 \mathrm{mcg} / \mathrm{kg}$, iv midazolam 30 $\mathrm{mcg} / \mathrm{kg}$ and iv Fentanyl $2 \mathrm{mcg} / \mathrm{kg}$ as premedication. Induction agent used was iv Propofol $3 \mathrm{mg} / \mathrm{kg}$, the end point being loss of eyelash reflex. Lubrication was done with thin layer of K-Y jelly over the back of cuff of i-gel/Classic LMA and device was introduced. After confirming the position of device, iv vecuronium $0.1 \mathrm{mg} / \mathrm{kg}$ was given. Patients were ventilated and anaesthesia was maintained with $\mathrm{O}_{2}, \mathrm{~N}_{2} \mathrm{O}$, iv Propofol and iv vecuronium with controlled ventilation. Diclofenac $75 \mathrm{mg}$ suppository was inserted for analgesia. At the end of surgery the device was removed after reversal with iv neostigmine $(50 \mathrm{mcg} / \mathrm{kg})+$ iv Atropine $(200$ $\mathrm{mcg} / \mathrm{kg}$ ). Hemodynamic parameters like pulse rate, non-invasive blood pressure, oxygen saturation were recorded pre-op, at induction, every five minutes intra-op and then post-operatively till one hour.

The i-gel or Classic LMA were inserted as recommended by manufacturer. The time between picking up the airway device and obtaining an effective airway was recorded. An effective airway is defined as normal thoraco-abdominal movement and a square wave capnograph trace. The number of insertion attempts was recorded for both i-gel/Classic-LMA. A failed attempt was defined as removal of the device from the mouth. Three attempts were allowed before device was considered as a failure. If an effective airway could not be achieved, one attempt with the other device was allowed. If an effective airway was not achievable with the alternate device (i-gel or Classic LMA) then the airway was achieved with endotracheal tube \& case was considered as failure \& documented. Gastric tube (12-14 no.) was inserted through the drainage tube of i-gel. The time taken to insert the gastric tube was recorded, and the placement was confirmed by synchronous injection of air and epigastric auscultation during apnea. In case of difficulty in introducing gastric tube two attempts were tried with the manipulation of the introducer. Inability to insert the gastric tube was recorded. Any adverse event was also noted.

At the end of the surgery after removing the airway device the $\mathrm{pH}$ of the dorsal surface of the cuff was noted with the sensitive litmus paper. Post operatively the patients were questioned directly about sore throat half an hour after admission to the recovery room. Sore throat incidence was evaluated using a 3 point scale as 2- continuous throat pain,1- throat discomfort, 0 - no complaints at all.

The statistical analysis for comparison was based on t test for time for effective airway (TFEA), hemodynamic variables, $\mathrm{pH}$ of the cuff. 2-tailed t test was used for number of attempts. Mann-Whitney test was used for the comparison of sore throat incidence.

\section{Results}

There was no difference in two groups with respect to demographic data and the surgical aspect.

\begin{tabular}{|c|c|c|c|}
\hline Weight & $\begin{array}{c}\text { i-gel } \\
(\mathrm{S} . \mathrm{D}=9.48\end{array}$ & $\begin{array}{c}\text { Classic LMA } \\
(\mathrm{S} . \mathrm{D}=9.12\end{array}$ & $\begin{array}{c}\mathrm{P}=0.809 \\
\text { Non-signif- } \\
\text { icant }\end{array}$ \\
\hline $\begin{array}{c}\text { Surgical } \\
\text { duration }\end{array}$ & $\begin{array}{c}69.60 \\
(\mathrm{~S} . \mathrm{D}=32.657)\end{array}$ & $\begin{array}{c}71.40 \\
(\mathrm{~S} . \mathrm{D}=32.195)\end{array}$ & $\begin{array}{c}\mathrm{P}=0.845 \\
\text { Non-signif- } \\
\text { icant }\end{array}$ \\
\hline Males & 12 & 13 & $50 \%$ \\
\hline Females & 13 & 12 & $50 \%$ \\
\hline
\end{tabular}

\begin{tabular}{|c|c|c|}
\hline Size & First Attempt & More than one attempt \\
\hline \multirow{2}{*}{3} & i-gel- 13 & i-gel-0 \\
\cline { 2 - 3 } & CLMA-5 & CLMA-7 \\
\hline \multirow{2}{*}{4} & i-gel-9 & i-gel- 3 \\
\cline { 2 - 3 } & CLMA-9 & CLMA-3 \\
\hline
\end{tabular}

As seen in this chart i-gel could be inserted in first attempt in 22 cases as against Classic LMA could be inserted in first attempt only in 14 cases. Only 3 cases of i-gel required second attempt for insertion as against 9 cases in Classic LMA which required second attempt for insertion. On one occasion Classic LMA needed 3 attempts for successful insertion and on one other occasion Classic LMA could not be inserted to achieve effective airway where the patient was intubated to continue the general anaesthesia. Success rate for insertion of i-gel was higher than Classic LMA. Mean of no. of attempts required for i-gel insertion was 1.12 and for Classic LMA was 1.45. More than one attempts required for i-gel in 3 cases and 10 cases for Classic LMA. This difference was statistically significant. Time required for achieving effective airway (TFEA) was shorter with i-gel than Classic LMA. It was statistically significant. (TFEA for i-gel - 20 seconds, TFEA for Classic LMA - 52 seconds). After the removal of the device at the end of the surgery $\mathrm{pH}$ of the cuffs for i-gel was 7.12 and for Classic LMA was 6.82. This was statistically not significant. Post operatively throat discomfort (Grade 1) was found in 5 cases of i-gel and 9 cases of Classic LMA. Throat pain (Grade 2) was found in 3 cases of Classic LMA. This was found statistically significant. 


\begin{tabular}{|c|c|c|c|}
\hline & i-gel & Classic LMA & \\
\hline Mean attempts & $1.12(\mathrm{~S} . \mathrm{D}=0.332)$ & $1.45(\mathrm{~S} . \mathrm{D}=0.588)$ & 2-tailed Test, significant \\
\hline Time For Effective Airway (TFEA) & $20.24(\mathrm{~S} . \mathrm{D}=22.908)$ & $52.458(\mathrm{~S} . \mathrm{D}=41.341)$ & $\mathrm{P}=0.002 \mathrm{t}$ Test, significant \\
\hline $\mathrm{pH}$ of the cuff secretions & $7.12(\mathrm{~S} . \mathrm{D}=0.53)$ & $6.82(\mathrm{~S} . \mathrm{D}=0.73)$ & $\mathrm{P}=0.1773 \mathrm{t}$ Test, not significant \\
\hline Sore throat scale (Mean rank) & 19 & 31.25 & $\mathrm{P}<0.05$ Mann Whitney Test, significant \\
\hline
\end{tabular}

Passing the NG tube was easy in most of the i-gel insertions with mean time of 9 seconds. Only in one case with i-gel NG tube could not be passed.

\begin{tabular}{|c|c|c|c|c|}
\hline & Minimum & Maximum & Mean & Std. Deviation \\
\hline TFRT (N=24) & 5 & 20 & 9.167 & 3.749 \\
\hline
\end{tabular}

\section{Adverse effects in study group}

\begin{tabular}{|c|c|c|c|}
\hline Sr.No & Complications & i-gel, N=25 & CLMA, N=25 \\
\hline 1 & $\begin{array}{c}\text { Inability of device } \\
\text { insertion }\end{array}$ & - & 1 \\
\hline 2 & Desaturation & - & - \\
\hline 3 & Laryngospasm & - & - \\
\hline 4 & Bronchospasm & - & - \\
\hline 5 & Gastric insufflations & - & - \\
\hline 6 & $\begin{array}{c}\text { Regurgitation of gastric } \\
\text { contents }\end{array}$ & - & - \\
\hline 7 & Blood stained secretions & - & 3 \\
\hline
\end{tabular}

\section{Discussion}

In our study we found that the success rate for introduction of i-gel in first attempt was higher (22/25) than classic LMA (14/25) and time required for effective airway establishment was shorter for i-gel ( 20.24 seconds) than Classic LMA( 52.458 ). To insert i-gel there was no need to insert finger in the oral cavity of the patient as was required in Classic LMA. No extra adjustments or techniques were required to insert $\mathrm{i}$-gel which might be the reason for the decreased time required $[25,28]$. This time difference was statistically significant. But for the routine cases which were preoxygenated before insertion of the device there was no episode of desaturation. It might be important in emergency situations where achieving the airway at the earliest is of prime importance as when the patients may desaturate faster. In this regard i-gel is found to be quicker and superior way of achieving the airway. I gel has been shown to be a superior airway device over Classic LMA in CPCR [30].

Use of LMA for positive pressure ventilation is not new but has some controversies [10] over it regarding risk of gastric regurgitation and pulmonary aspiration. i-gel appears to be superior to Classic LMA in this regard as it contains second channel for NG tube placement which may reduce the chances of regurgitation and aspiration. Optimal positioning of NG tube determines the correct position of i-gel. Some studies have defined position of the device by passing fibreoptic scope through it [28]. We did not pass fibrescope through the devices due to non-availability. NG tube could be passed through all the i-gels except one where it did not pass but effective ventilation was still achieved. NG tube insertion was easy in majority of the cases with average time of 9.167 seconds. Regurgitation of gastric contents was not observed from gastric channel of any of the i-gels. Also after removal of the device at the end of the surgery $\mathrm{pH}$ of the dorsal side of the cuff of both devices was checked with sensitive litmus paper. Acidic $\mathrm{pH}$ was not found in any of the cuffs. There was no statistical difference found between $\mathrm{pH}$ of cuffs of the devices. Brimacombe et al in previous cadaveric studies in other devices like LMA Proseal showed that inserting NG tube is always beneficial to prevent pulmonary aspiration. Gastric fluid was shown to bypass the pharynx and mouth when NG tube was open and airway device was in correct position [12]. The hemodynamic changes after insertion of both devices were statistically not significant. Fujii et al showed that Classic LMA provides smooth and safe emergence from anaesthesia with less hemodynamic changes 14 . Such studies for i-gel are not yet done. There were no episodes of desaturation, laryngospasm or bronchospasm. Post operatively throat discomfort ( Grade 1 ) was noted in 5 and 9 cases of i-gel and Classic LMA respectively and throat pain (Grade 2 ) was noted in 3 cases with Classic LMA. This was statistically significant. Visible blood was found on cuffs of 3 LMA Classic but not on any of i-gels. This shows that i-gel is less traumatic as compared to Classic LMA. Reiger et al concluded with regards to minor laryngo-pharyngeal morbidity that advantage of LMA Classic over endotracheal tube is questionable [15]. Sore throat and dysphagia are common after LMA insertion. Incidence of sore throat varies in different studies according to size, design of the airway device, lubricant used, cuff pressure of the device and \% of nitrous oxide used in the study. Generally this sore throat and dysphagia are transient in post-operative period [16] and i-gel appears to be the device which contributes very little to it.

\section{Conclusion}

To conclude, i-gel is an effective alternative supra-glottic airway device which is easier to insert than classic LMA and allows easy passage of naso-gastric tube through it. There is no blood staining of i-gel after removal and it is less traumatic to the pharyngeal tissues with very little incidence of post-operative sore throat.

\section{References}

[1]. Brain AI (1983) The laryngeal mask-a new concept in airway management. Br J Anaesth 55(8): 801-805.

[2]. Verghese C, Brimacombe JR (1996) Survey of laryngeal mask airway usage in 11,910 patients: Safety and efficacy for conventional and non-conventional usage. Anesth Analg 82(1): 129-133.

[3]. Devitt JH, Wenstone R, Noel AG, O’Donnell MP (1994) The laryngeal mask airway and positive-pressure ventilation. Anesthesiology 80(3): 550555.

[4]. Latorre F, Eberle B, Weiler N, Mienert R, Stanek A, et al. (1998) Laryngeal mask airway position and the risk of gastric insufflations. Anesth Analg 86(4): 867-871.

[5]. Brain AT, Verghese C, Strube PJ (2000) The LMA 'ProSeal--a laryngeal mask 
with an oesophageal vent. Br J Anaesth 84 (5): 650-654.

[6]. Brimacombe J, Keller C (2000) The ProSeal laryngeal mask airway: A randomized, crossover study with the standard laryngeal mask airway in paralyzed, anesthetized patients. Anesthesiology 93(1): 104-109.

[7]. Brain AIJ (1993) The Intavent Laryngeal Mask instruction manual.(3rd edtn) Maidenhead, Intavent Orthofix, UK.

[8]. Grady DM, McHardy F, Wong J, Jin F, Tong D, et al. (2001) Pharyngolaryngeal morbidity with the laryngeal mask airway in spontaneously breathing patients: does size matter ? Anesthesiology 94(5): 760-766.

[9]. Tan SM, Sim YY, Koay CK (2005) The ProSeal laryngeal mask airway size selection in male and female patients in an Asian population. Anaesth Intensive Care 33(2): 239-242.

[10]. Cook TM, JP Nolan, C Verghese, PJ Strube, M Lees, et al. ( 2002) Randomized crossover comparison of the ProSeal with the classic laryngeal mask airway in unparalysed anaesthetized patients. Br J Anaesth 88(4): 527-533.

[11]. Lu PP, Brimacombe J, Yang C, Shyr M (2002) ProSeal versus the Classic laryngeal mask airway for positive pressure ventilation during laparoscopic cholecystectomy. Br J Anaesth 88(6): 824-827.

[12]. Keller C, Brirnacombe J, Kleinsasser A, Loeckinger A (2000) Does the ProSeal laryngeal mask airway prevent aspiration of regurgitated fluid? Anesth Analg 91(4): 1017-1020.

[13]. Levitan RM, Kinkle WC (2005) Initial anatomical investigations of I-gel the novel supra-glottic airway device. Anaesthesia 60(10): 1022-1026.

[14]. Fujii Y, Toyooka H, Tanaka H (1997) Cardiovascular responses to tracheal extubation or LMA removal in normotensive and hypertensive patients. Can J Anaesth 44(10): 1082-1086.

[15]. Rieger A, Brunne B, Hass I, Brummer G, Spies C, et al. (1997) Laryngopharyngeal complaints following laryngeal mask airway and endotracheal intubation J Clin Anesth 9(1): 42-46.

[16]. Zimmert M, Zwirnert P, Kruse E (1999) Effects on vocal function and incidence of laryngeal disorder when using a laryngeal mask airway in comparison with an endotracheal tube. Eur J Anaesthesiol 16(8): 511-515.

[17]. Brain Al, McGhee TD, McAteer EJ, Thomas A, Abu-Saad MA, et al. (1985) The laryngeal mask airway. Development and preliminary trials of a new type of airway. Anaesthesia 40(4): 356-361.

[18]. P Barker, JA Langton, PJ Murphy, DJ Rowbotham (1992) Regurgitation Of Gastric Contents During General Anaesthesia Using The Laryngeal Mask Airway. Br J Anaesth 69(3): 314-315.
[19]. Wood ML, Forrest ET (1994) The haemodynamic response to the insertion of the laryngeal mask airway: a comparison with laryngoscopy and tracheal intubation. Acta 38(5): 510-513.

[20]. Imai M, Matsumura C, Hanaoka Y, Kemmotsu O (1995) Comparison of cardiovascular responses to airway management: fibreoptic intubation using a new adapter, laryngeal mask insertion, or conventional laryngoscopic intubation. J Clin Anesth 7(1): 14-18.

[21]. Brimacombe J (1995) The advantages of the LMA over the tracheal tube or facemask: a meta-analysis. Can J Anaesth 42(11): 1017-1023.

[22]. Brain AI, Verghese C, Strube P, Brimacombe J (1995) A new laryngeal mask prototype. Preliminary evaluation of seal pressures and glottic isolation. Anaesthesia 50(1): 42-48.

[23]. Asai T, Howell TK, Koga K, Morris (1998) Appropriate size and inflation of the laryngeal mask airway. Br J Anaesth 80(4): 470-474.

[24]. Brimacombe J, Keller C (1999) Laryngeal mask airway size selection in males and females: ease of insertion, oropharyngeal leak pressure, pharyngeal mucosal pressures and anatomical position. Br J Anaesth 82(5): 703-707.

[25]. Gatward JJ, Cook TM, Seller C (2008) Evaluation of i-gel no.4 in 100 nonparalysed patients. Anaesthesia 63(10): 1124-1132.

[26]. Cheong YP, Park SK, Son Y, Lee KC, Song YK, et al. (1999) Comparison of incidence of gastroesophageal reflux and regurgitation associated with timing of removal of LMA: on appearance of signs of rejection versus after recovery of conciosness. J Clin Anaesth 11(8): 657-665.

[27]. Miller RD (2005) Textbook of anaesthesia. (6 th edtn). Churchill Livingstone, New York, USA. 2: 3376.

[28]. Michalek P, Hodgkinson P, Donaldson W (2008) Fiberoptic intubation through an I-gel supraglottic airway in two patients with predicted difficult airway and intellectual disability. Anesthesia \& Analgesia 106(5): 15011504 .

[29]. Richez B, Saltel L, Banchereau F, Torrielli R, Cros AM (2008) A new single use supraglottic airway device with noninflatable cuff and an esophageal vent: an observational study of the i-gel. Anesth Analg 106(4): 1137-1139.

[30]. Gatward JJ, Thomas MJ, Nolan JP, Cook TM (2008) Effect of chest compressions on the time taken to insert airway devices in a manikin. Br J Anaesth 100(3): 351-356. 